\title{
ResearchOnline@JCU
}

This is the Accepted Version of a paper published in the journal: Clinical Cancer Research

Blake, Stephen J., Dougall, William C., Miles, John J.,

Teng, Michelle W.L., and Smyth, Mark J. (2016) Molecular pathways: targeting CD96 and TIGIT for cancer

Immunotherapy. Clinical Cancer Research, 22 (21). pp.

5183-5188.

http://dx.doi.org/10.1158/1078-0432.CCR-16-0933 


\section{Molecular Pathways: Targeting CD96 and TIGIT for Cancer}

\section{Immunotherapy}

Stephen J. Blake ${ }^{1 *}$, William C. Dougall ${ }^{2 *}$, John J. Miles ${ }^{3,4,5}$, Michele W. L. Teng ${ }^{1,5}$ and Mark J. Smyth ${ }^{2,5}$

${ }^{1}$ Cancer Immunoregulation and Immunotherapy, QIMR Berghofer Medical Research Institute, Herston, Queensland, Australia.

${ }^{2}$ Immunology in Cancer and Infection Laboratory, QIMR Berghofer Medical

Research Institute, Herston, Queensland, Australia.

${ }^{3}$ Human Immunity Laboratory, QIMR Berghofer Medical Research Institute, Herston, Queensland, Australia.

${ }^{4}$ Institute of Infection and Immunity, Cardiff University, Cardiff, UK.

${ }^{5}$ School of Medicine, University of Queensland, Herston, Queensland, Australia.

*These authors contributed equally

Corresponding Author: Mark J. Smyth, Immunology in Cancer and Infection Laboratory, QIMR Berghofer Medical Research Institute, 300 Herston Road, Herston 4006, Australia. PH: +61 (7) 3845 3957; E-mail: mark.smyth@qimrberghofer.edu.au

\section{Running title: CD96 and TIGIT in Cancer Immunotherapy}

The project was funded by a National Health and Medical Research Council of Australia (NH\&MRC) Project Grant (1088960) and Development Grant (1093566) a Cancer Council of Queensland (CCQ) Project Grant (1083776), and a Cancer Research Institute CLIP grant. M. J. S. is supported by a Senior Principal Research Fellowship (1078671).

Conflicts of Interest: M. J. S., and J.J.M. have been supported by a scientific research agreement with Bristol Myers Squibb. M. W. L. T. received an honorarium 
from Merck Sharp and Dohme. 


\begin{abstract}
The receptors CD96 and TIGIT are expressed on the surface of T and NK cells and recent studies suggest both play important inhibitory roles in immune function. CD96 has been shown to modulate immune cell activity in mice, with $C d 96^{-/-}$mice displaying hypersensitive NK cell responses to immune challenge and significant tumor resistance. TIGIT overexpression has been shown to reduce NK cell-mediated cytotoxicity. TIGIT is also upregulated on T-cells during cancer and chronic viral infection, with expression associated with effector T-cell exhaustion and increased Treg suppression. The counterbalance between the putative inhibitory CD96, TIGIT receptors and the activating receptor, CD226, offers unique strategies for immunooncology drug development. Blocking CD96 or TIGIT with monoclonal antibodies (mAbs) has been shown to improve tumor control in mice, in particular when used in combination with PD-1/PD-L1 blockade. These results have highlighted these pathways as promising new targets for immune modulation. This review will examine the rationale behind targeting CD96 and TIGIT and discuss the potential approaches in translating these preclinical findings into novel clinical agents.
\end{abstract}




\section{Background}

\section{CD155, CD226 and TIGIT in immune regulation and cancer}

The development of antibodies targeting immune checkpoint receptors PD-1 (1) and CTLA-4 (2) has been a monumental step forward in the clinical success of cancer immunotherapy. While highly successful as monotherapies, more than a dozen alternate pathways exist that modulate immune responses (3), suggesting combination approaches may greatly augment response rates. This hypothesis is supported by a recent clinical trial, demonstrating improved objective responses when PD-1 and CTLA-4 inhibitors were used in combination (4). While antibodies against CTLA-4 and PD-1 are thought to act predominately through T-cells, another immune cell type the natural killer (NK) cell is gaining traction as a target for cancer immunotherapy, particularly for the control of metastases and blood cancers (5). NK cells are part of the innate lymphocyte family and play a critical role in viral and tumor immune surveillance. NK cells act by detecting and killing infected or cancerous cells via perforin-mediated cytotoxicity and also regulate immune responses through the release of cytokines (6).

Candidate pathways for cancer immunotherapy include a cluster of immunoglobulin superfamily receptors that interact with nectin and nectin-like molecules (NECL), which are critical regulators in immune surveillance. Nectin and NECL family members were first characterized as adhesion molecules, mediating both homo- and heterophilic interactions (7). A diverse range of nectin and NECL proteins-receptor interactions exist, with roles in immune regulation, virus entry to cells and normal development $(7,8)$. Several nectin and NECL proteins have prominent roles in cancer 
surveillance, with CD155 (necl-5, PVR) the most well-characterized. While expression is low in normal tissue, CD155 is highly expressed on many cancer cell lines and primary tumors (9). CD155 has been linked with enhanced tumor proliferation (10) and migration (9). CD155 is also upregulated on many immune cells during inflammation (11) and on tumor-associated APCs (12). CD155 expression is thought to modulate T and NK cell responses through CD226, TIGIT and CD96 interactions (Table 1 and Figure 1).

Engagement between CD155 and CD226 or TIGIT has been a major focus of research, with CD226 having dual roles as both an activating and adhesion receptor on NK cells $(13,14)$, while TIGIT acts as an inhibitory receptor, shown to reduce NK cell cytokine production and cytotoxicity $(15,16)$. CD226 is also considered an activating receptor for $\mathrm{CD}^{+} \mathrm{T}$-cells (17) with its downregulation observed in advanced cancer and associated with T-cell exhaustion $(12,18)$. The role of CD226 in tumor immune surveillance is supported by accelerated tumor growth in $C d 226^{-/-}$ mice $(19,20)$. Conversely, TIGIT is highly upregulated on both $\mathrm{CD} 8^{+} \mathrm{T}$-cells and Tregs in many clinical tumor settings $(12,18,21)$, with expression also correlating with other immune checkpoints such as PD-1. Across in vitro human assays, primate models and mouse tumor models, TIGIT blockade has been shown to enhance T-cell function in particular, in combination with other checkpoints such as PD-1(12), PDL1 $(21,22)$ and TIM-3 (23). While CD155 is considered the dominant ligand for CD226 and TIGIT, CD226 can also interact with CD112 (24) and TIGIT can interact with CD112 and CD113 (25).

\section{CD96 expression, ligand interactions and putative signaling pathways}


CD96 (TACTILE) was first identified as an Ig superfamily receptor (26), however it is now known to be a member of the extended nectin/necl family and its role in immune function has received little attention until recently. CD96 expression is broadly similar between mice and humans, and is present on a proportion of hematopoietic stem cells, $\alpha \beta$ and $\gamma \delta$ T-cells, NK cells and a sub-population of B-cells in humans (27-31) and present on $\alpha \beta$ and $\gamma \delta$ T-cells, NK cells and NKT cells in mice $(11,32)$. CD96 is not expressed on other immune cells and expression is generally low or absent in organs without lymphocyte infiltrate (29). Of interest, CD96 is expressed at far higher levels in mice, with almost all cells positive for the receptor at resting state while basal expression is lower in humans (26). Interestingly, CD96 has been shown to be highly expressed in acute myeloid leukemia (AML), T-cell acute lymphoblastic leukemia (T-ALL) (29) and myelodysplastic syndromes (33). CD96 has additionally been proposed as a cancer stem cell marker in leukaemia $(30,33)$.

Akin to DNAM and TIGIT, the main ligand for CD96 is CD155, to which it binds with an affinity stronger than CD226, but weaker than TIGIT. Human CD96, CD226, TIGIT bind to CD155 with dissociation constants $\left(\mathrm{K}_{\mathrm{d}}\right)$ of $37.6 \mathrm{nM}, 119 \mathrm{nM}$ and 3.15 nM respectively (25). Of note, mouse CD96 (mCD96) but not human CD96 (hCD96) has been shown to bind CD111 (nectin-1) (32). Other key differences also exist between human and mice. For example, hCD96 exists as two splice variants that confer different binding affinities to CD155 (29). The sequence of hCD96 but not mCD96 contains a potential SH-2 domain binding-site within the cytoplasmic tail in the form of an YXXM motif (29), similar to that found in activating receptors. Both human and mouse CD96 sequences contain immunoreceptor tyrosine-based inhibitory motifs (ITIMs) (34) that putatively may provide inhibitory signals to lymphocytes 
following ligation. However, downstream signaling of the CD96 receptor has not been evaluated in detail. Given CD155 contains cytoplasmic signaling motifs, it will be of interest to determine whether CD96 engagement triggers reverse CD155 signaling. Indeed, TIGIT/CD155 ligation has been shown to modulate the function of CD155 expressing DC cells, inducing IL-10 secretion (25).

\section{Functions of CD96}

Initial investigations of CD96 biology suggested a role in mediating human NK cell adhesion to CD155 expressing target cells and was also proposed as a weak NK cell activating receptor (31). CD96 was also described as an adhesion molecule to CD155 and CD111 in mouse studies $(29,32)$. The first evidence that CD96 might be acting as an inhibitory receptor was shown in $C d 96^{-/}$mice, where NK cells produced greater IFN- $\gamma$ in responses to LPS, IL-12 or IL-18 stimulation (11). This study also demonstrated a role for CD96 in cancer immune surveillance, with $C d 96^{-/}$mice showing robust resistance to experimental lung metastases and MCA-induced

fibrosarcomas. The potential of targeting CD96 to enhance NK cell control of metastases was highlighted in our recent article (35). In this study, we demonstrated that mAbs against CD96 could reduce the number of lung metastases in a range of spontaneous and experimental models. The activity of anti-CD96 was dependent on NK cells, IFN- $\gamma$ and CD226.

\section{Clinical-Translational Advances}

\section{Therapeutic approaches}

The complexity of interaction dynamics within the CD96/TIGIT/CD226/CD155 axis poses both opportunities and challenges for therapeutic translation in oncology. At the 
most fundamental level, the net inhibitory signals from either TIGIT or CD96 are counterbalanced via multiple mechanisms by the activating signal of CD226. Thus, an understanding of the dynamic regulation of CD226 expression and activity must be a core consideration when attempting to modulate TIGIT and CD96 activity. Therapeutic antibodies that reduce co-inhibitory signaling via blockade of CD155 binding to CD96 and/or TIGIT have considerable experimental support in preclinical cancer models $(21,35)$. These data support a correlation between antibody blockade of the CD96/TIGIT/CD155 axis with enhanced anti-cancer activity through increased $\mathrm{CD}^{+}$T-cell or increased NK cell function for anti-TIGIT and anti-CD96, respectively. As an alternative to blockade of ligand binding to co-inhibitory receptors, antibodies that stabilize CD155 binding to CD226 might selectively potentiate an activating signal, and may serve as a therapeutic strategy by providing a more robust counterbalance to TIGIT and CD96.

Functional TIGIT suppression of anti-tumor responses is both intrinsic to effector Tcells and indirect, via enhancement of Treg activity. The enrichment of TIGIT expression on tumor-infiltrating Tregs compared to peripheral Tregs (23) would suggest that maximal anti-tumor response could be achieved via modification of the $\mathrm{mAb} F c$ region. Increased binding to activating FcR could mediate a selective depletion of $\mathrm{TIGIT}^{+}$Tregs at the tumor site through antibody dependent cellularcytotoxicity (ADCC) or -phagocytosis (ADCP), similar to what has been described for anti-CTLA-4 antibodies (36). The relatively greater TIGIT expression on Tregs versus T-effector cells within tumor site would optimally enable this approach. While CD96 is expressed on $\mathrm{CD}^{+}$T-cells (11), further work is necessary to determine whether CD96 influences the suppressive function of Tregs, and to define CD96 
expression on Tregs and Teff within the tumor microenvironment and periphery. Importantly, the ability of blocking CD96 mAb to reduce B16F10 lung metastases was not dependent on activating FcR (35), indicating that anti-tumor activity was not due to selective immune subset depletion.

Since receptor-binding domains are conserved in CD155 (25), therapeutic antibodies targeting this ligand are not likely to have selective effects on inhibitory (TIGIT and CD96) signals versus activating (CD226) pathways. However, the relatively higher binding affinity of CD155 to TIGIT or CD96 compared with the lower affinity CD226 interaction can theoretically be exploited for drug development. Greater selectivity towards CD226-dependent signaling and subsequent lymphocyte activation could be achieved using engineered variants (or "muteins") of CD155 or other nectins that retain TIGIT and/or CD96 binding but cannot bind CD226. By competing with native CD155, these modalities would functionally block TIGIT and/or CD96 inhibitory signaling but retain the activation through CD226. The ability of TIGIT to form a signaling-competent homodimer (37) or, conversely, a heterocomplex with CD226 which impairs CD226 activation signaling (21), reveals the dynamic interactions of this receptor system and other potential anti-cancer drug approaches. Antibodies that target the interface between TIGIT homodimers may reduce inhibitory signaling, while targeting interactions between TIGIT and CD226 extracellular domains might conceivably block heterocomplex formation and relieve TIGIT-mediated inhibition of CD226 signaling.

Preclinical mechanistic data and known lymphocyte expression patterns suggests that combination immunotherapy strategies targeting TIGIT and/or CD96 will have 
improved anti-tumor responses. For instance, combining anti-CD96 with either antiCTLA-4 or anti-PD-1 mAbs resulted in a greater reduction in B16F10 lung metastasis compared to monotherapy treatment, an effect dependent on NK cells (35). Moreover, survival of mice with 4T1.2 spontaneous metastases, was significantly increased by combining anti-CD96 and anti-CTLA-4 or anti-PD-1 compared to monotherapy. Similarly, treatment of CT26 tumors with a combination of anti-TIGIT and anti-PDL1 dramatically improved anti-tumor responses compared to each as a monotherapy (21), while using an anti-TIM-3 mAb in Tigit ${ }^{-/-}$mice improved the control of B16F10 metastases and subcutaneous tumors (23). There is also evidence that targeting TIGIT and CD96 in combination could be exploited as a therapeutic strategy. While Tigit ${ }^{-1}$ mice showed enhanced immunity to either B16F10 melanoma grown as subcutaneous tumors (23), experimental B16F10 lung metastasis were not reduced in Tigit $^{-/}$mice (11). However treatment of Tigit $^{-/}$mice with an anti-CD96 mAb resulted in a greater reduction of B16F10 or EO771 lung metastasis than that observed upon anti-CD96 $\mathrm{mAb}$ treatment in wild-type mice (35), suggesting that these pathways may instruct non-overlapping lymphocyte subsets and/or distinct molecular mechanisms.

While immunotherapy functions by increasing host anti-tumor immunity, the induction of immune-related adverse events (irAEs) in patients can limit certain approaches. Clinically, CTLA-4 blockade is associated with more high-grade irAEs than PD-1 (4), and in agreement, Ctla-4 $4^{-/-}$mice develop a lethal lymphoproliferative disorder (38). However, $P d-1^{-/-}$mice, can spontaneously develop a range of less severe immune pathologies $(39,40)$. To date, Tigit $^{-/-}(41)$ or Cd96 $^{-/}$mice $(11)$ have not shown spontaneous development of overt immune pathologies, however Tigit $^{-/}$ mice were more sensitive to the induction of experimental autoimmune 
encephalomyelitis (41) and TIGIT blockade was shown to increase experimental arthritis development (42). While caution in the over interpretation of animal studies is advised, these preclinical observations suggest CD96 or TIGIT blocking therapies may have favourable clinical toxicity profiles.

\section{Open questions and challenges}

Currently, the foremost challenge for translating TIGIT- or CD96-targeted therapies is to functionally validate blockade of these receptors in human lymphocytes. Two useful validation surrogates include the analysis of TIGIT and CD96 expression/function in patient tumor infiltrating lymphocytes (TILs), or on T-cells from chronic virus-infected individuals, and any correlation with exhaustion markers and/or phenotype. TIGIT was reportedly co-expressed with PD-1 on effector CD8 ${ }^{+} \mathrm{T}-$ cells during HIV or SIV infection and increased TIGIT expression correlated with disease progression (22). TIGIT levels were elevated on $\mathrm{CD} 4^{+}$and $\mathrm{CD} 8^{+}$TILs and coexpressed with $\mathrm{PD}-1$ on $\mathrm{CD} 8^{+} \mathrm{T}$-cells in NSCLC, colon cancer and melanoma samples $(12,21)$, with similar expression seen on PBMCs from AML patients (18). Promisingly, treatment of HIV-specific $\mathrm{CD} 8^{+}$T-cells with an anti-TIGIT mAb increased IFN $\gamma$ production (22). Similarly, treatment of tumor-specific melanoma $\mathrm{CD}^{+}$TILs with anti-TIGIT mAb augmented proliferation and IFN- $\gamma$ production (12, 43). These data are consistent with a co-inhibitory function for TIGIT in the context of chronic antigen stimulation in humans and provide a sound rationale for further development of TIGIT blockade therapeutics.

Currently, the validation of hCD96 as a potential immunotherapy target is not as advanced as TIGIT. While CD96 surface expression is increased on human T-cells 
post activation $(26,44)$, a high percentage of resting $\mathrm{CD}^{+} \mathrm{T}$-cells from healthy controls express CD96 and the fraction of $\mathrm{CD} 96^{+} \mathrm{CD} 8^{+} \mathrm{T}$-cells was reduced in HIVinfected individuals (44). Interestingly, serum levels of soluble CD96 were found to be elevated in patients with chronic viral hepatitis B infection (45), suggesting that persistent antigen exposure can increase CD96 levels and/or cell surface shedding. In human cancers, CD96 surface expression on TILs versus PBMCs has not been well characterized, however CD96 mRNA expression, along with TIGIT, was highly expressed and associated with a T-cell signature in lung cancer (21). Current data from preclinical cancer models indicates an inhibitory function for CD96 on NK cells, but whether the same function exists on mouse T-cells and human NK or T-cells remains to be elucidated. The determination of CD96 expression in human T-cell subsets, TILs and a comprehensive validation of functional pathway activity in human immune cells is an area of active research and are obligatory prerequisites for the development of CD96 blockade therapeutics.

Presently, the relationship between specific structural/biophysical attributes and optimal anti-cancer mechanisms of TIGIT- or CD96-targeted therapies are not well described. The rationale for blocking CD155/TIGIT binding to augment CD8 ${ }^{+}$T-cell function as a cancer immunotherapy approach is sound. While TIGIT robustly inhibits human NK cell-mediated cytotoxicity in vitro $(16,46)$, in mice, TIGIT NK cell-mediated tumor suppression is less pronounced than CD96 (11). Moreover, the potentially dominant contribution of TIGIT ${ }^{\text {high }}$ Treg cells in the anti-cancer immune response (23) suggests that a blocking antibody might also augment effector functions. These observations should be reconciled to understand the full, integrated 
contribution of TIGIT on distinct lymphocytes (eg $\mathrm{CD} 8^{+} \mathrm{T}$-cells, $\mathrm{CD} 4^{+} \mathrm{T}$-cells and NK cells) for an optimal anti-tumor response.

The ability of CD96 or TIGIT to counterbalance activation mediated by CD226 appears to drive most of the anti-cancer activities of CD96/TIGIT targeted therapies. Indeed, a CD226 blocking antibody reversed suppression of CT26 tumors by an antiTIGIT mAb (21). Similarly, CD96 suppression of B16F10 lung metastases, via genetic deletion or blocking antibody was mostly dependent on intact CD226 function $(11,35)$. Within this paired inhibitory/activating axis, the magnitude and integrated quality (activation versus inhibition) of lymphocyte signaling is dictated by the relative availability (and selective competitive binding) of certain ligands but also by the kinetics and coordinated expression of receptor levels. To this end, CD226 was down regulated on $\mathrm{CD}^{+}$T-cells and NK cells in $\mathrm{AML}$ patients $(18,47)$, and low CD226 was detected on CD8 ${ }^{+}$TILs from melanoma patients compared with PBMCs (12). CD226, TIGIT and CD96 receptor expression was reportedly dynamically modulated by ligand exposure. CD155 interaction decreases surface expression of CD226 (48) and CD96 (31) on contacting cells. Conversely, increased levels of CD226 and CD96 have been observed in $C d 155^{-/-}$mice, while TIGIT levels were unchanged (49). These dynamic alterations in activating and inhibitory receptor levels may tip the balance in net signaling output in a context-dependent manner and potentially alter responses to CD96- and TIGIT- targeted therapies.

Clearly, emerging preclinical evidence suggests there is much promise in modulating the CD96/TIGIT/DNAM/CD155 axis for immuno-oncology. A clearer understanding of the molecular- and context-dependent mechanisms by which CD96 and TIGIT 
function in immunity will pave the way for its therapeutic application in cancer, either as monotherapies or in combination with other therapies. At this stage, the ultimate impact of fine-tuning the function of TIGIT and CD96 receptors on cancer patient outcomes are wholly unknown but will be interesting to monitor as knowledge advances. 


\section{References}

1. Weber JS, D'Angelo SP, Minor D, Hodi FS, Gutzmer R, Neyns B, et al. Nivolumab versus chemotherapy in patients with advanced melanoma who progressed after anti-CTLA-4 treatment (CheckMate 037): a randomised, controlled, open-label, phase 3 trial. Lancet Oncol. 2015;16:375-84.

2. Hodi FS. Improved survival with ipilimumab in patients with metastatic melanoma. N Engl J Med. 2010;363:711-23.

3. Page DB, Postow MA, Callahan MK, Allison JP, Wolchok JD. Immune modulation in cancer with antibodies. Annu Rev Med. 2014;65:185-202.

4. Postow MA, Chesney J, Pavlick AC, Robert C, Grossmann K, McDermott D, et al. Nivolumab and ipilimumab versus ipilimumab in untreated melanoma. $\mathrm{N}$ Engl $\mathrm{J}$ Med. 2015;372:2006-17.

5. Childs RW, Carlsten M. Therapeutic approaches to enhance natural killer cell cytotoxicity against cancer: the force awakens. Nat Rev Drug Discov. 2015.

6. Vivier E, Tomasello E, Baratin M, Walzer T, Ugolini S. Functions of natural killer cells. Nat Immunol. 2008;9:503-10.

7. Samanta D, Almo SC. Nectin family of cell-adhesion molecules: structural and molecular aspects of function and specificity. Cell Mol Life Sci. 2015;72:645-58. 8. Martinet L, Smyth MJ. Balancing natural killer cell activation through paired receptors. Nat Rev Immunol. 2015;15:243-54.

9. Sloan KE, Eustace BK, Stewart JK, Zehetmeier C, Torella C, Simeone M, et al. CD155/PVR plays a key role in cell motility during tumor cell invasion and migration. BMC Cancer. 2004;4:73.

10. Kono T, Imai Y, Yasuda S, Ohmori K, Fukui H, Ichikawa K, et al. The $\mathrm{CD} 155 /$ poliovirus receptor enhances the proliferation of ras-mutated cells. Int $\mathrm{J}$ Cancer. 2008;122:317-24.

11. Chan CJ, Martinet L, Gilfillan S, Souza-Fonseca-Guimaraes F, Chow MT, Town L, et al. The receptors CD96 and CD226 oppose each other in the regulation of natural killer cell functions. Nat Immunol. 2014;15:431-8.

12. Chauvin JM, Pagliano O, Fourcade J, Sun Z, Wang H, Sander C, et al. TIGIT and PD-1 impair tumor antigen-specific CD8+ T cells in melanoma patients. J Clin Invest. 2015;125:2046-58.

13. Lakshmikanth T, Burke S, Ali TH, Kimpfler S, Ursini F, Ruggeri L, et al. NCRs and DNAM-1 mediate NK cell recognition and lysis of human and mouse melanoma cell lines in vitro and in vivo. J Clin Invest. 2009;119:1251-63.

14. Iguchi-Manaka A, Kai H, Yamashita Y, Shibata K, Tahara-Hanaoka S, Honda $\mathrm{S}$, et al. Accelerated tumor growth in mice deficient in DNAM-1 receptor. J Exp Med. 2008;205:2959-64.

15. Stanietsky N, Rovis TL, Glasner A, Seidel E, Tsukerman P, Yamin R, et al. Mouse TIGIT inhibits NK-cell cytotoxicity upon interaction with PVR. Eur J Immunol. 2013;43:2138-50.

16. Stanietsky N, Simic H, Arapovic J, Toporik A, Levy O, Novik A, et al. The interaction of TIGIT with PVR and PVRL2 inhibits human NK cell cytotoxicity. Proc Natl Acad Sci U S A. 2009;106:17858-63.

17. Shibuya A, Campbell D, Hannum C, Yssel H, Franz-Bacon K, McClanahan T, et al. DNAM-1, a novel adhesion molecule involved in the cytolytic function of $\mathrm{T}$ lymphocytes. Immunity. 1996;4:573-81.

18. Kong Y, Zhu L, Schell TD, Zhang J, Claxton DF, Ehmann WC, et al. T-Cell Immunoglobulin and ITIM Domain (TIGIT) Associates with CD8+ T-Cell 
Exhaustion and Poor Clinical Outcome in AML Patients. Clin Cancer Res. 2016;22:3057-66.

19. Guillerey C, Ferrari de Andrade L, Vuckovic S, Miles K, Ngiow SF, Yong $\mathrm{MC}$, et al. Immunosurveillance and therapy of multiple myeloma are CD226 dependent. J Clin Invest. 2015;125:2077-89.

20. Gilfillan S, Chan CJ, Cella M, Haynes NM, Rapaport AS, Boles KS, et al. DNAM-1 promotes activation of cytotoxic lymphocytes by nonprofessional antigenpresenting cells and tumors. J Exp Med. 2008;205:2965-73.

21. Johnston RJ, Comps-Agrar L, Hackney J, Yu X, Huseni M, Yang Y, et al. The immunoreceptor TIGIT regulates antitumor and antiviral CD8(+) T cell effector function. Cancer Cell. 2014;26:923-37.

22. Chew GM, Fujita T, Webb GM, Burwitz BJ, Wu HL, Reed JS, et al. TIGIT Marks Exhausted T Cells, Correlates with Disease Progression, and Serves as a Target for Immune Restoration in HIV and SIV Infection. PLoS Pathog. 2016;12:e1005349.

23. Kurtulus S, Sakuishi K, Ngiow SF, Joller N, Tan DJ, Teng MW, et al. TIGIT predominantly regulates the immune response via regulatory $\mathrm{T}$ cells. J Clin Invest. 2015;125:4053-62.

24. Bottino C, Castriconi R, Pende D, Rivera P, Nanni M, Carnemolla B, et al. Identification of PVR (CD155) and Nectin-2 (CD112) as cell surface ligands for the human DNAM-1 (CD226) activating molecule. J Exp Med. 2003;198:557-67.

25. Yu X, Harden K, Gonzalez LC, Francesco M, Chiang E, Irving B, et al. The surface protein TIGIT suppresses $\mathrm{T}$ cell activation by promoting the generation of mature immunoregulatory dendritic cells. Nat Immunol. 2009;10:48-57.

26. Wang PL, O'Farrell S, Clayberger C, Krensky AM. Identification and molecular cloning of tactile. A novel human $\mathrm{T}$ cell activation antigen that is a member of the Ig gene superfamily. J Immunol. 1992;148:2600-8.

27. Garg S, Madkaikar M, Ghosh K. Investigating cell surface markers on normal hematopoietic stem cells in three different niche conditions. Int J Stem Cells. 2013;6:129-33.

28. Toutirais O, Cabillic F, Le Friec G, Salot S, Loyer P, Le Gallo M, et al. DNAX accessory molecule-1 (CD226) promotes human hepatocellular carcinoma cell lysis by Vgamma9Vdelta2 T cells. Eur J Immunol. 2009;39:1361-8.

29. Meyer D, Seth S, Albrecht J, Maier MK, du Pasquier L, Ravens I, et al. CD96 interaction with CD155 via its first Ig-like domain is modulated by alternative splicing or mutations in distal Ig-like domains. J Biol Chem. 2009;284:2235-44.

30. Hosen N, Park CY, Tatsumi N, Oji Y, Sugiyama H, Gramatzki M, et al. CD96 is a leukemic stem cell-specific marker in human acute myeloid leukemia. Proc Natl Acad Sci U S A. 2007;104:11008-13.

31. Fuchs A, Cella M, Giurisato E, Shaw AS, Colonna M. Cutting edge: CD96 (tactile) promotes NK cell-target cell adhesion by interacting with the poliovirus receptor (CD155). J Immunol. 2004;172:3994-8.

32. Seth S, Maier MK, Qiu Q, Ravens I, Kremmer E, Forster R, et al. The murine pan T cell marker CD96 is an adhesion receptor for CD155 and nectin-1. Biochem Biophys Res Commun. 2007;364:959-65.

33. Zhang W, Shao Z, Fu R, Wang H, Li L, Liu H. Expressions of CD96 and CD123 in Bone Marrow Cells of Patients with Myelodysplastic Syndromes. Clin Lab. 2015;61:1429-34.

34. McVicar DW, Burshtyn DN. Intracellular signaling by the killer immunoglobulin-like receptors and Ly49. Sci STKE. 2001;2001:re1. 
35. Blake SJ, Stannard K, Liu J, Allen S, Yong MC, Mittal D, et al. Suppression of Metastases Using a New Lymphocyte Checkpoint Target for Cancer

Immunotherapy. Cancer Discov. 2016;6:446-59.

36. Selby MJ, Engelhardt JJ, Quigley M, Henning KA, Chen T, Srinivasan M, et al. Anti-CTLA-4 antibodies of IgG2a isotype enhance antitumor activity through reduction of intratumoral regulatory T cells. Cancer Immunol Res. 2013;1:32-42.

37. Stengel KF, Harden-Bowles K, Yu X, Rouge L, Yin J, Comps-Agrar L, et al. Structure of TIGIT immunoreceptor bound to poliovirus receptor reveals a cell-cell adhesion and signaling mechanism that requires cis-trans receptor clustering. Proc Natl Acad Sci U S A. 2012;109:5399-404.

38. Waterhouse P, Penninger JM, Timms E, Wakeham A, Shahinian A, Lee KP, et al. Lymphoproliferative disorders with early lethality in mice deficient in Ctla-4. Science. 1995;270:985-8.

39. Nishimura H, Okazaki T, Tanaka Y, Nakatani K, Hara M, Matsumori A, et al. Autoimmune dilated cardiomyopathy in PD-1 receptor-deficient mice. Science. 2001;291:319-22.

40. Nishimura H, Nose M, Hiai H, Minato N, Honjo T. Development of lupus-like autoimmune diseases by disruption of the PD-1 gene encoding an ITIM motifcarrying immunoreceptor. Immunity. 1999;11:141-51.

41. Joller N, Hafler JP, Brynedal B, Kassam N, Spoerl S, Levin SD, et al. Cutting edge: TIGIT has T cell-intrinsic inhibitory functions. J Immunol. 2011;186:1338-42.

42. Levin SD, Taft DW, Brandt CS, Bucher C, Howard ED, Chadwick EM, et al. Vstm3 is a member of the CD28 family and an important modulator of T-cell function. Eur J Immunol. 2011;41:902-15.

43. Inozume T, Yaguchi T, Furuta J, Harada K, Kawakami Y, Shimada S. Melanoma Cells Control Antimelanoma CTL Responses via Interaction between TIGIT and CD155 in the Effector Phase. J Invest Dermatol. 2016;136:255-63. 44. Eriksson EM, Keh CE, Deeks SG, Martin JN, Hecht FM, Nixon DF. Differential expression of CD96 surface molecule represents CD8(+) T cells with dissimilar effector function during HIV-1 infection. PLoS One. 2012;7:e51696. 45. Gong J, Zhu C, Zhuang R, Song C, Li Q, Xu Z, et al. Establishment of an enzyme-linked immunosorbent assay system for determining soluble CD96 and its application in the measurement of sCD96 in patients with viral hepatitis B and hepatic cirrhosis. Clin Exp Immunol. 2009;155:207-15.

46. Liu S, Zhang H, Li M, Hu D, Li C, Ge B, et al. Recruitment of Grb2 and SHIP1 by the ITT-like motif of TIGIT suppresses granule polarization and cytotoxicity of NK cells. Cell Death Differ. 2013;20:456-64.

47. Sanchez-Correa B, Gayoso I, Bergua JM, Casado JG, Morgado S, Solana R, et al. Decreased expression of DNAM-1 on NK cells from acute myeloid leukemia patients. Immunol Cell Biol. 2012;90:109-15.

48. Seth S, Qiu Q, Danisch S, Maier MK, Braun A, Ravens I, et al. Intranodal interaction with dendritic cells dynamically regulates surface expression of the costimulatory receptor CD226 protein on murine T cells. J Biol Chem. 2011;286:3915363.

49. Nagumo Y, Iguchi-Manaka A, Yamashita-Kanemaru Y, Abe F, Bernhardt G, Shibuya A, et al. Increased CD112 expression in methylcholanthrene-induced tumors in CD155-deficient mice. PLoS One. 2014;9:e112415.

50. Lozano E, Dominguez-Villar M, Kuchroo V, Hafler DA. The TIGIT/CD226 axis regulates human T cell function. J Immunol. 2012;188:3869-75. 
51. Joller N, Lozano E, Burkett PR, Patel B, Xiao S, Zhu C, et al. Treg cells expressing the coinhibitory molecule TIGIT selectively inhibit proinflammatory Th1 and Th17 cell responses. Immunity. 2014;40:569-81. 
Table 1. Biological roles of TIGIT and CD96 in lymphocyte function and outcomes of pathway inhibition relevant to immunotherapy

\begin{tabular}{|c|c|c|}
\hline & TIGIT & CD96 \\
\hline T-cells & $\begin{array}{l}\text { Increased T-effector cell function in } \\
\text { tigit }^{-/-} \text {knockout or anti-TIGIT mAb } \\
\text { treated mice, increased human T- } \\
\text { effector function following antibody } \\
\text { treatment or shRNA knockdown } \\
\text { REFS (12, 21, 22, 41, 50) } \\
\text { Reduced suppressive function of Treg } \\
\text { cells; reduced suppression of anti- } \\
\text { tumor immune responses by tigit }{ }^{-/-} \\
\text {Tregs, increased suppression of Th1, } \\
\text { Th17 immune responses by TIGIT } \\
\text { compared to TIGIT- Tregs in mice } \\
\text { REFS (23, 51) } \\
\text { Associated with markers of T-cell } \\
\text { exhaustion in tumors or chronic viral } \\
\text { infections, blockade improves effector } \\
\text { T-cell function REFS (12, 18, 21, 22, } \\
\text { 43) }\end{array}$ & $\begin{array}{ll}\text { - } & \text { Role of CD96 in T-cell function currently } \\
\text { unknown } & \text { Surface expression of CD96 upregulated } \\
\text { on activated human T-cells REF (26) } \\
\text { - } \\
\text { CD96 mRNA expression increased and } \\
\text { associated with a T-cell signature in non- } \\
\text { squamous non-small cell lung cancer } \\
\text { cohort REF (21) } \\
\text { - Reduced expression on CD8+ T-cells } \\
\text { from chronic HIV infected patients } \\
\text { compared to healthy controls REF (44) }\end{array}$ \\
\hline $\begin{array}{l}\text { NK } \\
\text { cells }\end{array}$ & 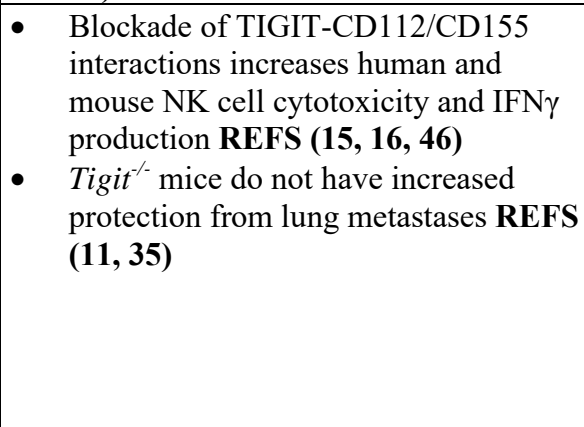 & $\begin{array}{ll}\text { - } & \text { Putative adhesion molecule of mouse and } \\
\text { - } & \text { human NK cells REFS }(\mathbf{2 9}, \mathbf{3 1}, \mathbf{3 2}) \\
\text { Putative activating receptor for human NK } \\
\text { cells REF (31) } \\
\text { - } C d 96^{-/-} \text {mice or blockade with anti-CD96 } \\
\text { mAb increases NK production of IFN } \gamma \\
\text { REFS }(\mathbf{1 1 , 3 5 )} \\
\text { - } C d 96^{-/-} \text {mice or blockade with anti-CD96 } \\
\text { mAb increases control of NK cell- } \\
\text { dependent tumors and metastases REFS } \\
(\mathbf{1 1 , 3 5 )}\end{array}$ \\
\hline
\end{tabular}


Figure 1. The regulation of inhibitory versus activation signals in NK cell and T-cells by CD96/TIGIT/CD226 receptors is achieved by complex receptor/ligand and receptor/receptor counterbalancing mechanisms. CD155 and CD96, TIGIT, CD226 are all members of the Ig superfamily and all share similar variable or constant Ig motifs in the extracellular domains, single-pass transmembrane regions and short cytoplasmic domains.

A). CD155 levels on different cells are upregulated as a result of cellular stress. In cancer, CD155 is increased on transformed cells and antigen presenting cells (APC) within the tumor microenvironment and is sensed by the CD96/TIGIT/CD226 receptors to modulate anti-tumor immunity.

B). Upon exposure to increasing levels of CD155, the net activation or inhibition of lymphocytes is fine-tuned by the integrated signalling of CD96, TIGIT and CD226. This net integration is dictated by the relative binding affinity of CD155 for different receptors, relative abundance of activation (CD226) vs. inhibitory (CD96, TIGIT) receptors, the strength and quality of signal transduction by each receptor and the modulation of ligand binding and biochemical signal transduction by homo- and heterodimerization of receptor complexes.

The cytoplasmic domain of CD226 has a tyrosine (Y322) and serine (S329), which become phosphorylated in a CD155-dependent manner. Phosphorylation of Y322 confers binding of CD226 to the SH2-domain containing protein Grb2 and downstream signalling. Serine 329 phosphorylation of CD226 mediates activation of protein kinase $\mathrm{C}$ and the association with lymphocyte function-associated antigen 1 (LFA1) as an intermediate for further signal transduction. TIGIT contains an immunoreceptor tyrosine inhibitory motif (ITIM) domain and an immunoglobulin tail tyrosine (ITT) motif within the cytoplasmic tail. Upon ligand binding, both the ITT and ITIM domains of TIGIT are phosphorylated and recruit adaptor and signaling molecules. While both mCD96 and hCD96 contain an ITIM-like domain, the human CD96 cytoplasmic domain uniquely also includes a YXXM motif. A detailed review of CD226, TIGIT and CD96 signaling mechanisms is described in (8).

C). These molecular regulatory mechanisms provide distinct opportunities for drug development in immuno-oncology. 1). Therapeutic antibodies that block CD155 binding to CD96 or TIGIT would reverse the inhibitory signaling by these receptors. Using either anti-CD96 or anti-TIGIT mAbs, this approach has received specific experimental support in mouse cancer models. 2). Antibody or mutated ligand ("mutein") modalities which allow preferential signaling through the activating receptor CD226 might tip the balance between CD155-dependent activation and inhibition. For instance, a $\mathrm{mAb}$ that recognizes and stabilizes the unique interaction complex of CD155/CD226 could enhance stimulatory signaling through CD226.

Alternatively, engineered mutations in CD155 muteins that preferentially bind CD96 and/or TIGIT might competitively inhibit native CD155 binding and enhance lymphocyte activation. 3). The blockade of TIGIT/CD226 heterodimer or TIGIT/TIGIT homodimer by drug modalities would potentially reduce TIGITdependent inhibitory signals. 
Figure 1:

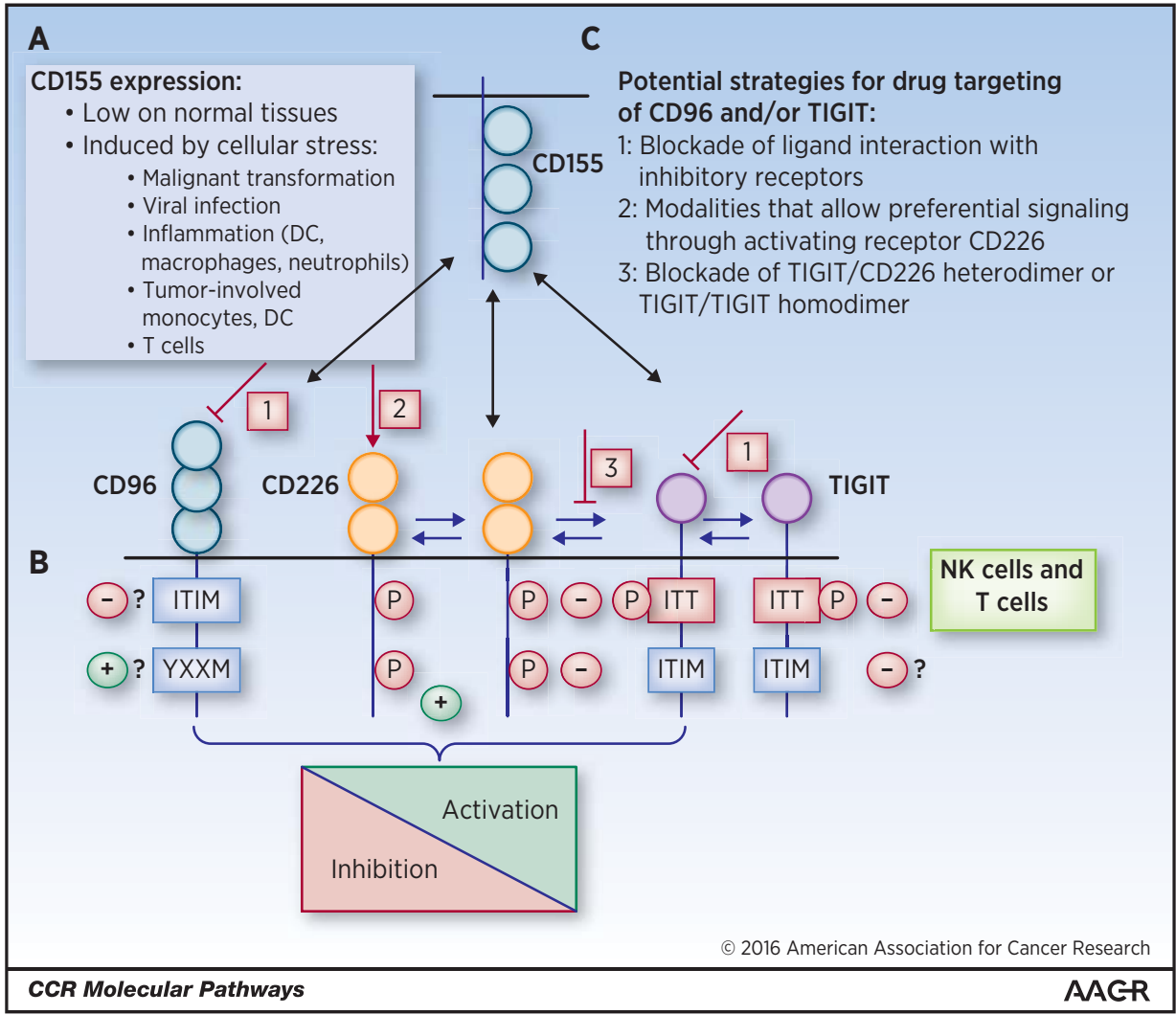

